\title{
Competency-based education in general surgery: Are Canadian residents ready?
}

\author{
Gabrielle Gauvin, MD, MS \\ Kathryn Hay, MD \\ Wilma Hopman, MA \\ Scott Hurton, MD, MSc \\ Stephanie Lim, MD \\ Boris Zevin, MD, PhD \\ Diederick Jalink, MD \\ Sulaiman Nanji, MD, PhD
}

Accepted Nov. 5, 2020

This paper was presented at the Canadian Surgery Forum.

\section{Correspondence to: \\ S. Nanji \\ Burr 2 Room 076 \\ Kingston General Hospital \\ 76 Stuart St \\ Kingston ON K7L 2V7 \\ sulaiman.nanji@kingstonhsc.ca}

Cite as: Can J Surg 2021 September 1; 64(5). doi: $10.1503 /$ cjs. 011520

\section{SUMmaRY}

Competency-based education (CBE) is currently being implemented by the Royal College of Physicians and Surgeons of Canada across all residency programs. This shift away from time-based residency is proposed to be the answer to maximize training opportunity in the era of work hour restrictions and growing concerns regarding accountability in medical education. A Web-based survey was conducted to obtain feedback from Canadian general surgery residents on their experience and perception of competence within core procedures, as well as attitudes toward CBE. A total of 244 residents completed the survey. For most procedures, more than $50 \%$ of residents felt they could perform the procedure with no guidance after completing 11-30 cases. Generally, residents were welcoming of CBE; however, medium-sized programs reported some concerns regarding inadequate exposure to cases and risk of training less well-rounded surgeons. This is valuable resident feedback for programs to consider during the implementation process. 
rating scale was proposed; the Perceived Procedural Competence Scale was used to describe the amount of guidance residents felt they needed for each procedure, ranging from needing guidance for most of the procedure to being ready for independent practice.

A total of 244 residents completed the entire survey for a response rate of $41.1 \%$. Results showed that laparoscopic appendectomies and cholecystectomies were the procedures that residents reported the most experience performing. Perceived competence for $50 \%$ of residents was achieved with 11-30 procedures for elective laparoscopic cholecystectomy, mastectomy, inguinal hernia repair, LAR, laparoscopic inguinal hernia and a right hemicolectomy (Figure 1). Perceived competence was also 11-30 cases for Hartmann procedure, open right hemicolectomy, LAR, and laparoscopic inguinal hernia repair; however, more than $50 \%$ of residents completed these procedures fewer than 10 times, indicating that several residents are not meeting case volume numbers where perceived competency is reported. Junior residents (postgraduate year [PGY] 1-2) performed 1-10 cases of laparoscopic appendectomy, laparoscopic cholecystectomy and open inguinal hernia repair. More than $60 \%$ of junior residents did not perform any bowel and breast cases. Senior residents (PGY 3-4) and chief residents (PGY5) completed 31-50 cases of laparoscopic appendectomy, laparoscopic cholecystectomy and open inguinal hernia repairs. Senior and chief residents reported performing 1-10 cases of right hemicolectomy, Hartmann procedure, LAR, mastectomy, SLNB, and laparoscopic inguinal hernia.

Compared with junior residents, chief residents reported that quantifying the number of performed procedures is not an adequate measure of competency. With CBE, each resident will need to demonstrate competence in specific procedures before transition to independent practice. This will potentially eliminate some of the variability in exposure to the breath of surgical procedures by different residents.

Large programs (> 37 residents) were most in favour of implementation of CBE, whereas mediumsized programs (26-36 residents) were most concerned about risk of training less well-rounded surgeons and poor exposure to cases. This concern may arise from the fact that medium-sized programs reported being the most satisfied with their current curriculum.

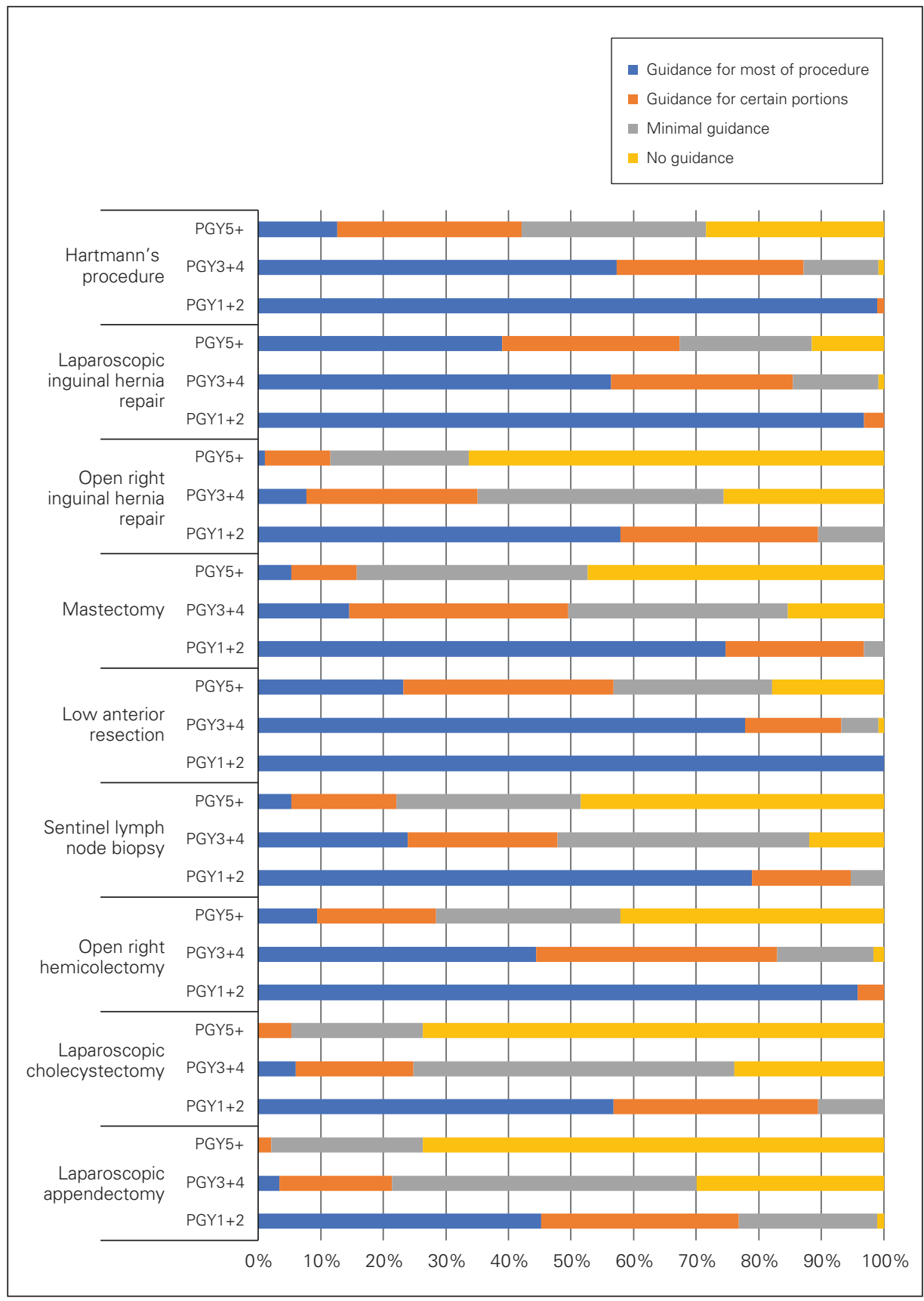

Fig. 1. Resident-perceived competence for 9 common general surgery procedures. $P G Y=$ postgraduate year. 
Compared with participants in larger programs, those in small programs ( $<25$ residents) felt that their academic half-days did not have clear objectives and did not provide essential information. Residents from small and large programs also reported there were too many noneducational activities. Addressing these concerns could ease resident fears about CBE implementation and help frame this transition in a positive light. During their CBE implementation in orthopedic surgery, the University of Toronto prioritized reducing repetitive activities and placing more focus on educational activities relating to residents' milestones, which was well received. ${ }^{4}$

Numerous evaluation and assessment methods are currently used by residency programs to track resident progress. In Canada, the main standardized performance evaluations for general surgery residents are the Interim Evaluation Reports (ITER) and the Final InTraining Evaluation Report (FITER)/Comprehensive Competency Report (CCR). A recent Canadian publication outlined the lack of assessment methods for specific general surgery skills such as endoscopy. ${ }^{5}$ In our survey, residents did not think their assessments were informing them of their competency, and they felt that a greater number of assessments in the operating room would be beneficial. Seventy-five per cent of participants believed they would not be able to finish their residency in less than 5 years. Eighty-seven per cent were receptive to taking the RCPSC exam in their fourth year of residency, allowing their last year to be rich in hands-on experience to facilitate a smoother transition to practice. When asked about the importance of written exams, chief residents rated them as less important than the junior residents. Senior residents provided a significantly higher rating than junior residents with regards to the importance of having assessors in the operating room, observing cases performed by residents.

\section{Conclusion}

The implementation of CBE introduces an opportunity to improve assessments and inform faculty of the needs of learners. In a CBE program, formative assessments of resident performance are completed multiple times per week and include assessments of performance in the operating room. However, 2 of the perceived barriers to frequent assessments include faculty buy-in and assessment burnout. ${ }^{1}$ Broad support from staff and consensus regarding definitions of competency for each procedure will be needed and have been shown to facilitate faculty engagement during transitioning to CBE. ${ }^{4}$ Postimplementation, it would be pertinent to evaluate how attitudes toward the CBE curriculum have changed and whether residents are satisfied with the new assessment methods.

Affiliations: From the Department of Surgery, Queen's University, Kingston, Ont. (Gauvin, Hay, Zevin, Jalink, Nanji); the Department of Public Health Sciences, Queen's University, Kingston, Ont. (Hopman); the Department of Surgery, University of Manitoba, Winnipeg, Man. (Hurton, Lim); and the Department of Surgery, CISSS de l'Outaouais, Gatineau, Que. (Gauvin).

Funding: The study was funded internally by the Department of Surgery, Queen's University.

Competing interests: None declared.

Contributors: All authors contributed substantially to the conception, writing and revision of this article and approved the final version for publication.

Content licence: This is an Open Access article distributed in accordance with the terms of the Creative Commons Attribution (CC BYNC-ND 4.0) licence, which permits use, distribution and reproduction in any medium, provided that the original publication is properly cited, the use is noncommercial (i.e., research or educational use), and no modifications or adaptations are made. See: https://creativecommons. org/licenses/by-nc-nd/4.0/

\section{References}

1. Frank JR, Snell LS, Cate OT, et al. Competency-based medical education: theory to practice. Med Teach 2010;32:638-45.

2. Bell RH, Biester TW, Tabuenca A, et al. Operative experience of residents in US general surgery programs: A gap between expectation and experience. Ann Surg 2009;249:719-24.

3. Jaffer U, Cameron AE. Laparoscopic appendectomy: a junior trainee's learning curve. 7SLS 2008;12:288-91.

4. Alman BA, Furguson P, Kraemer W, et al. Competency-based education: a new model for teaching Orthopedics. AAOS. Instr Course Lect 2013;62:565-9.

5. Delisle M, Chernos C, Park J, et al. Canadian general surgery residents' need formal curricula and objective performance assessments in gastrointestinal endoscopy training: a program director census. Surg Endosc 2018;32:5012-20. 